\title{
Orbital Floquet engineering of exchange interactions in magnetic materials
}

\author{
Swati Chaudhary, ${ }^{*}$ David Hsieh, and Gil Refael \\ Institute of Quantum Information and Matter, Department of Physics, California Institute of Technology, Pasadena, California 91125, USA
}

(Received 3 June 2019; published 9 December 2019)

\begin{abstract}
We present a scheme to control the spin-exchange interactions by manipulating the orbital degrees of freedom using a periodic drive. We discuss two different protocols for orbital Floquet engineering. In one case, a periodic drive modifies the properties of the ligand orbitals which mediate magnetic interactions between transition-metal ions. In the other case, we consider drive-induced mixing of $d$ orbitals on each magnetic ion. We first find that an AC Stark shift of orbitals induces a change comparable to that induced from photoinduced hopping schemes, but expands the applicable frequency ranges. Next, we find that radiatively induced coherent vibrations provide a realistic path for Floquet orbital engineering with short pulses of electric fields weaker than $0.5 \mathrm{~V} / \AA$ producing $5 \%-10 \%$ changes in the magnetic coupling of Mott insulators such as the rare-earth titanates.
\end{abstract}

DOI: 10.1103/PhysRevB.100.220403

Periodic drive is emerging as an intriguing tool for controlling and manipulating quantum many-body systems. Floquet engineering has been invoked in contexts ranging from the generation of artificial gauge fields to the realization of many-body localization [1-45] with ultracold atoms in optical lattices. These methods can potentially provide an external control knob for material properties, and can be naturally applied to controlling quantum materials $[46,47]$.

Recent works [48-55] discussed using Floquet engineering to manipulate magnetic exchange interactions. These schemes relied on properties of the photoassisted hopping and become effective only for electric fields well above $E \approx 1 \mathrm{~V} / \AA$. They feature a renormalized electronic hopping, and therefore renormalized energy splittings in the effective Floquet Hamiltonian. These works assume direct hopping between two magnetic ions, and we refer to them as the photomodified direct hopping scheme henceforth.

In transition-metal (TM) compounds, ligand ions are crucial for spin-exchange processes. For example, in twodimensional (2D) transition-metal trichalcogenides (TMTCs), the magnetic interactions are mainly mediated by ligand ions through multiple spin-exchange channels. The orbitals of these ligand ions provide extra degrees of freedom that can be manipulated to modify the exchange interactions. The magnetic coupling induced via ligand ions depends on the electronic energy and the shape of the orbitals available for spin exchange on the ligands, as well as on the strong orbitalspin interplay of the TM ions [56-61]. Many previous works have successfully manipulated some orbital properties using strain [62] and heterostructuring [63,64].

In this Rapid Communication, we propose a scheme to modify the exchange interactions by manipulating the orbital degrees of freedom with a periodic drive. Particularly, we consider changes in the hybridization and energy of the levels involved in exchange processes. We use two toy models where a strong time-dependent electric field couples two orbitals, either of the ligand ion or of the TM ion. While these two approaches which concentrate on a direct radiative effect serve to develop our ideas, we find that realizing the metalorbital manipulation scheme with radiatively induced phonons achieves significant improvements. It can be implemented by using an ultrashort midinfrared laser pulse, as opposed to a continuous wave in other schemes. Furthermore, this scheme extends the target frequency window to a range from $100 \mathrm{meV}$ to $10 \mathrm{eV}$, and in certain cases, a significant change can be observed at $E \approx 0.5 \mathrm{~V} / \AA$.

Floquet engineering with ligand orbitals. The spinexchange interactions between two metal ions $(M)$ are typically mediated by nonmagnetic intermediary ligand ions $(X)$ as shown in Fig. 1. This superexchange occurs due to virtual hopping of electrons within the cluster $M-X-M$. Therefore, the exchange interactions also depend on the properties of the nonmagnetic ions' orbitals, and can be controlled by manipulating the properties of the relevant ligand orbitals.

The effect of dynamically coupled ligand orbitals on the spin-exchange interactions follows from the Autler-Townes (AT) effect [65], where a periodic drive splits absorption peaks by changing the energy of the excited states. Similarly, ligandorbital mixing changes the energy and hybridization of virtual excitations. These, in turn, alter the exchange interactions mediated by the ligand atoms. Consider a simple toy model with two metal ions, each with one spin, and a ligand ion with two filled orbitals, which gives rise to an AFM interaction between metal-ion spins. Without a drive, the Hamiltonian includes hopping between ligand orbitals (subscript $\alpha$ ) and metal sites (subscript $i$ ), metal-ion on-site spin interactions, and the energy of the ligand orbitals. It is given by

$$
H_{1}=H_{0}+H_{t}=\sum_{\alpha=A, B} \sum_{\sigma} E_{\alpha} n_{\alpha \sigma}+U \sum_{i} n_{i \uparrow} n_{i \downarrow}+H_{t},
$$

and $H_{t}$, the metal sites to ligand-orbital hopping, is

$$
H_{t}=-\sum_{i} \sum_{\alpha} t_{\alpha} c_{\alpha \sigma}^{\dagger} c_{i \sigma}+\text { H.c. }
$$




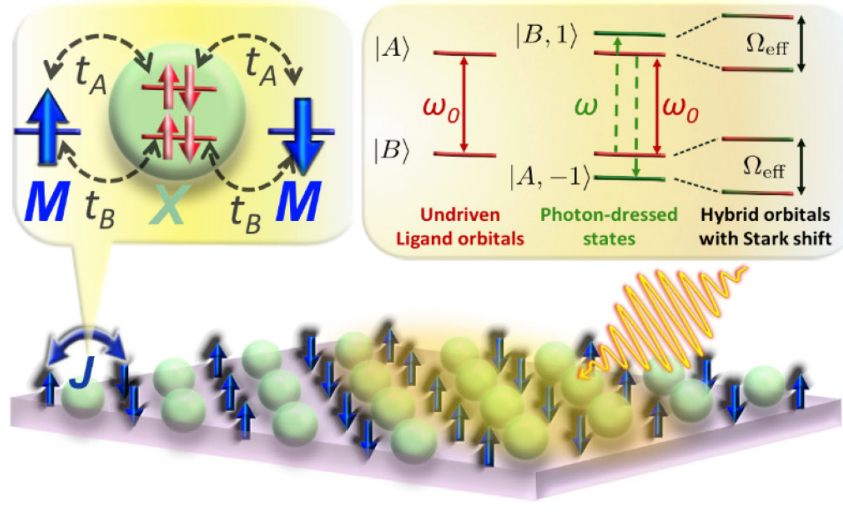

FIG. 1. Floquet engineering of spin-exchange interactions using ligand orbitals: Spin-exchange interactions are typically mediated by nonmagnetic ligand ions. Left panel: Virtual hopping of electrons from one magnetic ion $(M)$ to another via two orbitals $(A$ and $B$ ) of the ligand ion $(X)$. The magnetic-coupling strength depends on the hopping parameter and the energy of the orbitals involved in this hopping process. Right panel: In the presence of a periodic drive, these orbitals are replaced by hybridized photon-dressed orbitals (Floquet replicas shown in green). This splits the exchange channels and shifts the energies of virtual excitations, which modifies the exchange interactions.

with $\left|t_{\alpha}\right| \ll\left|E_{\alpha}\right|, U$. Assuming completely filled ligand orbitals, and a single spin per metal site on average, to find the exchange energy, we need fourth-order perturbation theory involving all possible exchange pathways (e.g., see Fig. 1 of the Supplemental Material [66]). The magnetic coupling $\left(J_{\mathrm{ex}}\right)$ up to fourth order is

$$
\begin{aligned}
J_{\mathrm{ex}}= & 4 \sum_{\alpha=A, B} t_{\alpha}^{4}\left(\frac{1}{\Delta_{\alpha}^{2} U}+\frac{1}{\Delta_{\alpha}^{3}}\right)+\frac{8 t_{A}^{2} t_{B}^{2}}{\Delta_{A} \Delta_{B} U} \\
& +4 t_{A}^{2} t_{B}^{2}\left(\frac{1}{\Delta_{A} \Delta_{B} \Delta_{A B}}+\frac{1}{\Delta_{A}^{2} \Delta_{A B}}+\frac{1}{\Delta_{B}^{2} \Delta_{A B}}\right),
\end{aligned}
$$

where $\Delta_{\alpha}=U-E_{\alpha}$ is the charge transfer gap and $\Delta_{A B}=$ $\left(\Delta_{A}+\Delta_{B}\right) / 2$. In Mott insulators, $\Delta_{\alpha} \gg U$, and thus the exchange interactions reduce to

$$
J_{\mathrm{ex}} \approx 4 \frac{t_{\mathrm{eff}}^{2}}{U}
$$

with $t_{\text {eff }}=\sum_{\alpha} \frac{t_{\alpha}^{2}}{\Delta_{\alpha}}$ the effective metal-ion ligand hopping.

Next, consider a drive coupling two ligand-ion orbitals,

$$
H(t)=\Omega e^{-i \omega t} c_{A \sigma}^{\dagger} c_{B \sigma}+\Omega^{*} e^{i \omega t} c_{B \sigma}^{\dagger} c_{A \sigma} .
$$

An oscillating electric field $\mathbf{E}(t)$, e.g., could couple orbitals $A$ and $B$ with strength $\Omega=\mathbf{E} \cdot \mathbf{P} / 2$, where $\mathbf{P}=e\langle A|\mathbf{r}| B\rangle$. This would modify orbitals involved in the spin exchange (Fig. 1) and thus change the energies of virtual excitations, as well as increase their number (while their weights still sum up to the same value as the undriven case-see Supplemental Material [66]).

The complete Hamiltonian, $H=H_{0}+H_{t}+H(t)$, can be analyzed using an extended Floquet basis, i.e., the tensor product of the electronic states and the harmonic ("photon") number $n$. We treat the hopping part $H_{t}$ perturbatively, with eigenstates of the Floquet Hamiltonian describing $H_{0}+H(t)$

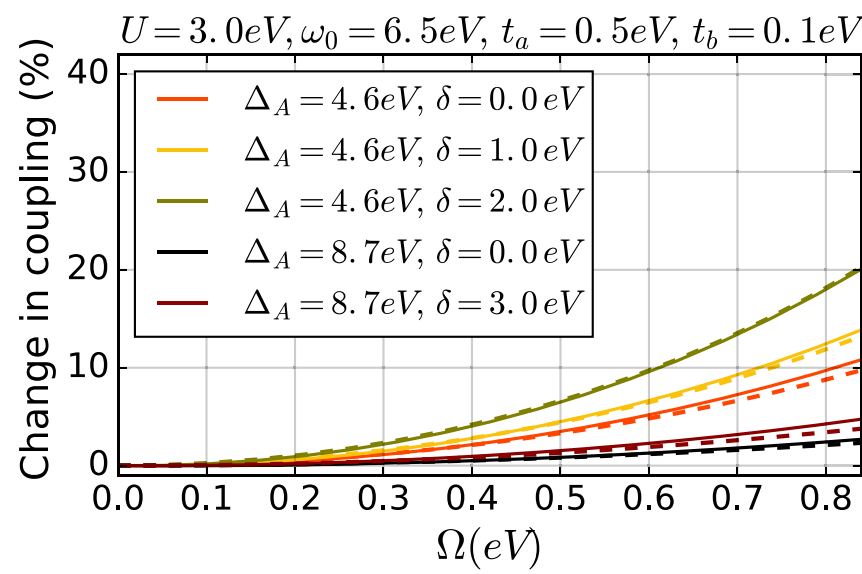

FIG. 2. Change in magnetic coupling vs drive strength $\Omega$ from numerics (solid lines) and theory (dashed lines) where the periodic drive mixes two orbitals of the ligand ion. The effect of the drive is large when the effective Rabi frequency is comparable to the charge transfer gap $\Delta_{A}$. These parameters were chosen according to the typical values of interaction energy $U$ and hopping parameter for TMTCs.

as virtual excitations. We choose drive parameters such that the effective-spin Hamiltonian picture remains valid. The periodic drive in Eq. (5) mixes the ligand orbitals $A$ and $B$, and the virtual excitations now involve

$$
\begin{aligned}
|P, n\rangle & =\cos \frac{\theta}{2}|A, n\rangle+\sin \frac{\theta}{2}|B, n+1\rangle, \\
|M, n\rangle & =\sin \frac{\theta}{2}|A, n\rangle-\cos \frac{\theta}{2}|B, n+1\rangle,
\end{aligned}
$$

where $\cos \theta=\frac{\delta}{\sqrt{\delta^{2}+4 \Omega^{2}}}, \sin \theta=-\frac{2 \Omega}{\sqrt{\delta^{2}+4 \Omega^{2}}}, \delta=\omega-\omega_{0}$ is the detuning, $n$ denotes the photon index, and $\omega_{0}=E_{B}-E_{A}$ is the energy difference between two ligand orbitals.

The drive-modified magnetic-coupling strength again emerges from fourth-order perturbation theory or numerical diagonalization of the Floquet Hamiltonian (truncated to include four Floquet zones). The expression for the new magnetic coupling $J_{\text {ex }}$ is similar to that in Eq. (3), with orbitals $A$ and $B$ replaced by their hybrid counterparts $|P, n\rangle$ and $|M, n\rangle$ [66]. How much $J_{\text {ex }}$ is modified is shown in Fig. 2. The exchange interactions depend mainly on $\Omega / \Delta_{A i}$ and $\delta / \Delta_{A i}$. The energy and number of virtual excitations change due to the splitting of spin-exchange channels into different Floquet sectors (right panel of Fig. 1). Significant changes in the coupling strength require a Rabi splitting $\Omega_{\text {eff }}$ between two states in each Floquet sector of the same order as the charge transfer gap $\Delta_{A i}$. The hopping amplitudes and the energy of these virtual levels now depend on detuning $\delta$. A large detuning brings some virtual-excitation levels close to the $d$ orbitals, which amplifies the effect (see Supplemental Material [66]).

As shown in Fig. 2, significant magnetic-coupling changes occur only if the shift in energy of virtual-excitation levels is comparable to the charge-transfer gap. Usually, the charge transfer gap $\Delta_{A i} \approx 5-10 \mathrm{eV}$, and thus a change of 5\%-10\%, requires $\Omega \approx 1 \mathrm{eV}$. A common ligand is sulfur, with accessible $3 s$ and $3 p$ orbitals. The energy difference between the two orbitals is $\omega_{0} \approx 10 \mathrm{eV}$ [67], and the dipole moment matrix 


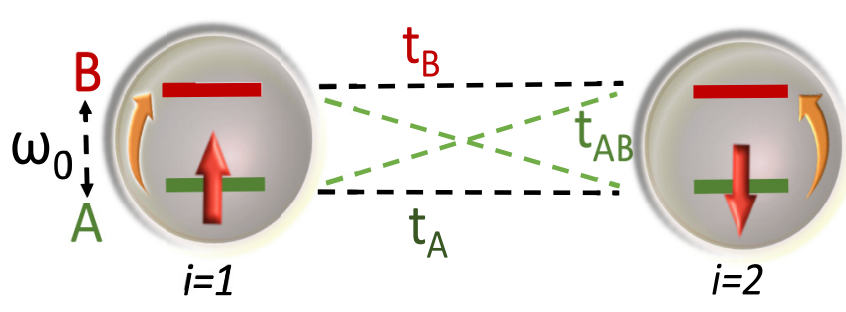

FIG. 3. Metal-orbital Floquet engineering: A two-site FermiHubbard model with two orbitals on each site at quarter filling. Two orbitals denoted by $A$ and $B$ with energy $E_{A}$, and $E_{A}+\omega_{0}$ are mixed using a periodic drive given in Eq. (11). For simplicity, we assume direct hopping between the metal ions.

element is $|\mathbf{P}| \approx 0.6 e \AA$ (see Supplemental Material [66]), and thus we need $E \approx 2 \mathrm{~V} / \AA$ to get $\Delta J / J \approx 10 \%$. Materials with a small charge transfer gap, large dipole moment matrix element, and small energy gap $\left(\omega_{0}\right)$ are ideal candidates for this scheme to work at a lower electric field. This charge transfer gap is usually lower for heavier transition-metal ions and decreases down the chalcogen group, which indicates that the materials with $\mathrm{Mn}, \mathrm{Ni}, \mathrm{Cu}$, and ligand $\mathrm{S}$ or $\mathrm{Se}$ would be the best candidates. However, a very high frequency is necessary as the $s-p$ energy separation is high, and for common ligands such as $\mathrm{O}$ and $\mathrm{S}$ it is above $10 \mathrm{eV}$, which makes it somewhat impractical.

Floquet engineering with metal-ion orbitals. The highfrequency requirement above goes away if we focus on metal orbitals. Above we assumed only a single orbital for each TM ion. Magnetism in TMs, however, is significantly affected by the occupancy of other $d$ orbitals, crystal-field splitting, and the on-site interactions. The $d$-orbital energies are usually split by the crystal field, yielding energy gaps from a few $\mathrm{meV}$ to almost $1 \mathrm{eV}$. A periodic drive can also modify these orbitals, and result in an AC Stark shift of both singly and doubly occupied sectors. Since the virtual excitations involve the Floquet orbitals, the magnetic coupling changes as long as the Stark shift is different for the low-energy and virtually excited states (see Supplemental Material [66]).

The effect of orbital mixing can be studied with a toy model where magnetic interactions arise from direct hopping between two TM ions. We consider a two-site Fermi-Hubbard model with two orbitals on each site and at quarter filling, and a periodic drive which couples the two levels on each site as shown in Fig. 3. Consider the Hamiltonian

$$
H=H_{t}+H_{k}+H_{0},
$$

where $H_{t}$ is a hopping term given by

$$
H_{t}=-\sum_{\sigma, \alpha=A, B} t_{\alpha} c_{1 \alpha \sigma}^{\dagger} c_{2 \alpha \sigma}-t_{A B} \sum_{\sigma, i \neq j} c_{1 A \sigma}^{\dagger} c_{2 B \sigma}+\text { H.c. }
$$

$H_{k}$ is the on-site Kanamori interaction [68],

$$
\begin{aligned}
H_{k}= & U \sum_{i, \alpha} \hat{n}_{i \alpha \uparrow} \hat{n}_{i \alpha \downarrow}+U_{1} \sum_{i, \alpha<\beta, \sigma, \sigma^{\prime}} \hat{n}_{i \alpha \sigma} \hat{n}_{i \beta \sigma^{\prime}} \\
& -J_{H} \sum_{i, \alpha<\beta, \sigma, \sigma^{\prime}} c_{i \alpha \sigma}^{\dagger} c_{i \alpha \sigma^{\prime}} c_{i \beta \sigma^{\prime}}^{\dagger} c_{i \beta \sigma},
\end{aligned}
$$

and the on-site energy

$$
H_{0}=\sum_{i} E_{A}\left(\hat{n}_{i A}-1\right)+\left(E_{A}+\omega_{0}\right) \hat{n}_{i B},
$$

with $U, U_{1} \gg t_{\alpha}$. At quarter filling, if $\omega_{0} \gg \frac{t_{\alpha}^{2}}{U}$, then the low-energy subspace consists of states with one spin in each $A$ orbital, and the magnetic coupling is approximately $J_{\mathrm{ex}}=$ $4 t_{A}^{2} / U$. On the other hand, if $\omega_{0}=0$ and $t_{a b}=0$, the ground state is ferromagnetic (FM) in spin but antiferromagnetic (AFM) in the orbital degree of freedom. We focus on the first scenario, which allows us to mix two orbitals by applying a periodic drive of the form

$$
H(t)=\sum_{i, \sigma}\left(\Omega e^{i \omega t} c_{i A \sigma}^{\dagger} c_{i B \sigma}+\Omega^{*} e^{-i \omega t} c_{i B \sigma}^{\dagger} c_{i A \sigma}\right) .
$$

Let us next focus on the Floquet eigenstates connected to the low-energy subspace of the undriven Hamiltonian, and where the effective spin picture is valid. We then calculate the spin-exchange interactions from the singlet-triplet energy splitting (details in the Supplemental Material [66]). As in Fig. 4, the magnetic-coupling strength strongly depends on the drive's frequency and strength. It is mainly the hybridization between $A$ and $B$ orbitals which affects the change. This hybridization depends on $\frac{\Omega}{\delta}$ and allows virtual excitation to states at energy $U_{1}+J$ in the doubly occupied sector. This contribution decreases as the detuning $\delta$ increases. A large detuning also lowers the energy of the virtual-excitation state at $U_{1}+J_{H}$ in the doubly occupied sector, but this is negligible compared to the hybridization effect as long as $\mid U_{1}+J_{H}-$ $U \mid \gg \delta$. The magnetic properties in this scheme are controlled by the ratios $\frac{\Omega}{\delta}, \frac{U_{1}+J_{H}}{U}$, and $\frac{t_{b}}{t_{a}}$.

This scheme requires magnetic materials where TM ions have a $d^{1}$ configuration. In transition-metal compounds with octahedral or tetrahedral ligand cages, $d$ orbitals split into $e_{g}$ and $t_{2 g}$ levels with crystal-field splitting parameter in the range of $0.3-1.5 \mathrm{eV}$. The periodic drive can be realized with an AC electric field which couples these $d$ orbitals. Therefore, the drive amplitude is $\Omega=e\left\langle\psi_{A}|\mathbf{E} \cdot \mathbf{r}| \psi_{B}\right\rangle / 2$. Only $d$ orbitals are involved in this transition, however, and dipole transitions between same-parity orbitals are forbidden. Nevertheless, the crystal field can induce $d-p$ mixing in noncentrosymmetric compounds, which lends some $p$ character to otherwise pure $d$ orbitals, and allows weak dipole transitions. For some tetrahedral complexes, this mixing is $1 \%-5 \%$ [69], and thus the matrix element $\left|\left\langle d_{i}|\mathbf{r}| d_{j}\right\rangle\right| \approx 0.05 e \AA$ which corresponds to a drive strength $\Omega \approx 0.02 \mathrm{eV}$ at $E=1 \mathrm{~V} / \AA$. Although there are materials with a tetrahedral ligand arrangement [70], currently we are not aware of any such magnetic materials where the TM ion with a $d^{1}$ configuration is surrounded by a tetrahedral cage of ligand ions. Nevertheless, in octahedral geometry, some mechanisms such as coupling with vibrational modes, and mixing with ligand $p$ orbitals [69,71-73] allow these $d-d$ transitions. This $d-p$ mixing can be estimated from the oscillator strength of $d$ - $d$ transitions in octahedral complexes (Table I of Ref. [72]), and it is roughly $0.1 \%$. This corresponds to a $d-d$ electric dipole moment matrix element, $P=e\left|\left\langle d_{t_{2 g}}|\mathbf{r}| d_{t_{e_{g}}}\right\rangle\right| \approx$ $0.01 e \AA$, and thus the drive strength, $\Omega \approx 0.005 \mathrm{eV}^{2}$ for $E=1 \mathrm{~V} / \AA$. 

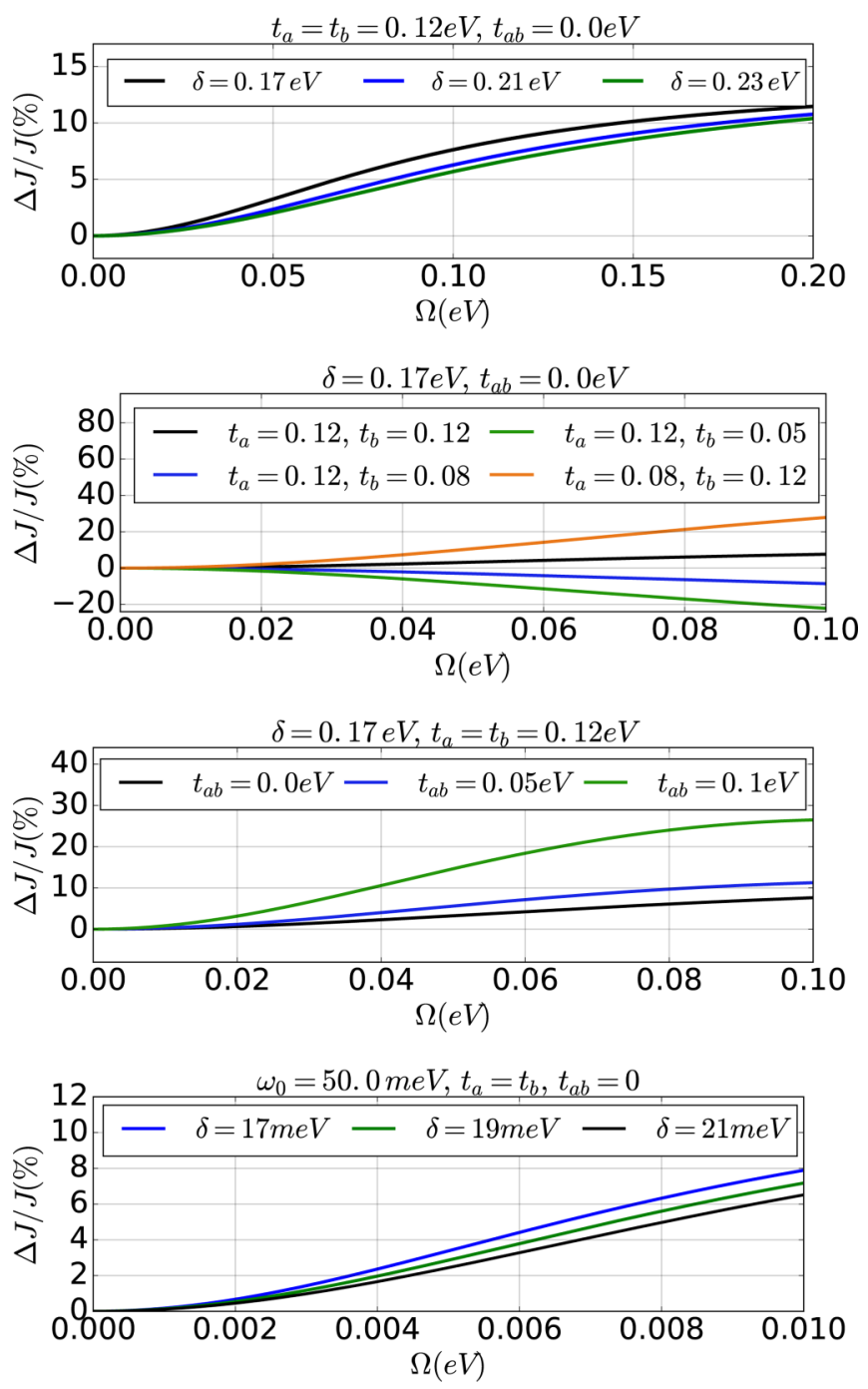

FIG. 4. Effect of different parameters on the change in magneticcoupling strength as a function of drive amplitude $\Omega$ for $U=4.0 \mathrm{eV}$, $J_{H}=0.8 \mathrm{eV}, U_{1}=U-2 J_{H}$, and $\omega_{0}=0.91 \mathrm{eV}$ when not specified. These changes are large when the detuning is decreased. The second panel shows that a large imbalance between $t_{a}$ and $t_{b}$ makes these changes more prominent. Similarly, we also observe that large $t_{a b}$ results in larger changes, and the change is proportional to $\Omega / \delta$ when $t_{a}=t_{b}$. In the last panel, we show the changes for a very small $\omega_{0}$ and small detuning where a significant change can be seen at extremely small drive amplitudes which is the case for a phonon drive.

The metal-orbital-hybridization scheme requires mixing the relevant two $d$ orbitals with light. In addition to the dipole transition, such mixing can also be achieved by employing two-photon processes. For a two-photon process between two $3 d$ orbitals, the drive amplitude, $\Omega \approx e^{2} E^{2} P_{d d}$, where $P_{d d} \approx$ $\frac{1}{2} \frac{|\langle 3 d|\mathbf{r}| 4 p\rangle / 2|^{2}}{E_{4 p}-E_{3,}} \approx 10^{-3} \AA^{2} / \mathrm{eV}$, and thus $\Omega \approx 10^{-3} \mathrm{eV}$ for the electric field, $E \approx 1 \mathrm{~V} / \AA$.

The applicability of Floquet orbital engineering, however, becomes much broader by employing direct vibrational coupling between the metal-ion $d$ levels. Contrary to the above hard-to-realize schemes, using coherent lattice vibrations to achieve a similar hybridization between two $d$ orbitals appears experimentally accessible. In per- fect octahedral symmetry, the direct vibrational coupling between some $d$ orbitals can occur for those Raman active modes which involve metal-ligand bond rotation. With typical phonon frequencies of such modes in the range $50-100 \mathrm{meV}$, it might be applied to materials with $d$ orbital energy splitting in the same range. This scheme can be used in some rare-earth titanates $\left(R \mathrm{TiO}_{3}\right)$, where even $t_{2 g}$ bands are nondegenerate with a crystal-field splitting $\Delta_{\mathrm{CF}} \approx 30-400 \mathrm{meV}$ [74], and some phonon modes [e.g., $\left.A_{g}(2), A_{g}(4), B_{1 g}(3), B_{1 g}(4), B_{2 g}(4)\right]$ which involve bond rotations have frequencies in the range $10-100 \mathrm{meV}[75,76]$. In this scheme, the drive strength is $\Omega \approx 0.25 u_{0} \mathrm{eV}$ (Sec. V of Supplemental Material [66]) where $u_{0}$ is the phonon-induced lattice displacement in $\AA$.

How lattice displacement $u_{0}$ depends on the electric field amplitude $E$ depends on different material properties and the details of the drive [77]. Here, we consider the indirect excitation of Raman modes in the impulsive limit which relies on large nonlinear coupling with some infrared (IR) active modes [78,79]. In these schemes, a phonon amplitude of approximately $0.03 \AA$ can be achieved by using a few-femtosecond mid-IR laser pulse with a maximum electric field amplitude $E \approx 0.5 \mathrm{~V} / \AA$ if the nonlinear coupling between two modes is sufficiently large, as is the case for Raman mode $A_{g}(25)$ and IR mode $B_{1 u}(54)$ in some Mott insulating titanates $[78,79]$ (more details in Supplemental Material Sec. VI [66]). This corresponds to a change of magnetic coupling by $5 \%-10 \%$. For some materials such as bismuth ferrites $\left(\mathrm{BiFeO}_{3}\right)$, the effect could be even more staggering, and combining Raman mode excitation schemes can lead to displacements of $0.05 \AA$ at $E<0.1 \mathrm{~V} / \AA ̊$ [77]. Such strong effects could arise in some $R \mathrm{TiO}_{3}$, but finding appropriate materials would require firstprinciples calculations which are beyond the scope of this Rapid Communication.

The coherent phonon scheme also offers the advantage of using ultrashort laser pulses. Short laser pulses (e.g., $50 \mathrm{fs}$ ) could produce lattice vibrations that persist for over $10 \mathrm{ps}$. This lowers the required fluence further as compared to other schemes where a laser with electric field amplitude $E \approx 1 \mathrm{~V} / \AA$ must be kept on for the entire duration of the measurement.

Conclusions. We proposed a protocol for controlling the magnetic properties of materials through Floquet engineering of their orbital degrees of freedom. While previous works [48-53] concentrated on the spin-exchange interaction change due to photoassisted hopping, we explore the effects of driveinduced modifications of the orbitals involved in the virtual processes involved in the exchange process.

We first developed our ideas by considering an AC Stark shift of excited levels in ligand orbitals as well as in the magnetic metal ions. Disappointingly, the AC Stark schemes yield significant changes with electric field amplitudes similar to those needed for the photomodified direct hopping schemes, $E \approx 1-5 \mathrm{eV} / \AA$, and require a continuous laser input. The frequency ranges of these various methods, however, are different. The ligand scheme needs $\omega \approx 5$ $10 \mathrm{eV}$ while the photomodified direct hopping works well for $\omega \approx 0.5-2 \mathrm{eV}$.

Orbital Floquet engineering using phonons, however, emerges as a very promising magnetic control scheme. Using 
short light pulses to induce coherent lattice vibrations can modify the magnetic metal-ion orbitals, and thereby change magnetic couplings by $5 \%-10 \%$ at much smaller frequencies $(\omega \approx 0.1 \mathrm{eV})$, and fields $E \approx 0.5 \mathrm{~V} / \AA$, an order of magnitude smaller than the amplitudes required for other schemes, and without the need for a continuous wave beam. Note that previous studies considered the phonon-induced magneticcoupling effects due to the shift in the atomic equilibrium position [79]. Also, Refs. [54,55] studied the effect of orbital properties in a photoassisted hopping scheme. We expanded both of these perspectives by considering orbital hy- bridization, and showed that controlling the orbital degrees of freedom with light opens up different possibilities for the manipulation of correlated quantum materials, and brings the quest for drive-controlled magnetism much closer to an experimental realization.

Acknowledgments. We acknowledge support from the IQIM, an NSF physics frontier center funded by the Gordon and Betty Moore Foundation. We are grateful for support from ARO MURI W911NF-16-1-0361 "Quantum Materials by Design with Electromagnetic Excitation" sponsored by the US Army.
[1] A. Eckardt, Rev. Mod. Phys. 89, 011004 (2017).

[2] T. Oka and H. Aoki, Phys. Rev. B 79, 081406(R) (2009).

[3] T. Kitagawa, T. Oka, A. Brataas, L. Fu, and E. Demler, Phys. Rev. B 84, 235108 (2011).

[4] J.-i. Inoue and A. Tanaka, Phys. Rev. Lett. 105, 017401 (2010).

[5] N. H. Lindner, G. Refael, and V. Galitski, Nat. Phys. 7, 490 (2011).

[6] N. Goldman and J. Dalibard, Phys. Rev. X 4, 031027 (2014).

[7] P. Titum, E. Berg, M. S. Rudner, G. Refael, and N. H. Lindner, Phys. Rev. X 6, 021013 (2016).

[8] M. Ezawa, Phys. Rev. Lett. 110, 026603 (2013).

[9] E. Bairey, G. Refael, and N. H. Lindner, Phys. Rev. B 96, 020201(R) (2017).

[10] D. Fausti, R. Tobey, N. Dean, S. Kaiser, A. Dienst, M. C. Hoffmann, S. Pyon, T. Takayama, H. Takagi, and A. Cavalleri, Science 331, 189 (2011).

[11] J. Klinovaja, P. Stano, and D. Loss, Phys. Rev. Lett. 116, 176401 (2016).

[12] A. G. Grushin, A. Gómez-León, and T. Neupert, Phys. Rev. Lett. 112, 156801 (2014).

[13] P. Hauke, O. Tieleman, A. Celi, C. Ölschläger, J. Simonet, J. Struck, M. Weinberg, P. Windpassinger, K. Sengstock, M. Lewenstein, and A. Eckardt, Phys. Rev. Lett. 109, 145301 (2012).

[14] F. Harper and R. Roy, Phys. Rev. Lett. 118, 115301 (2017).

[15] Y. Baum, E. P. van Nieuwenburg, and G. Refael, SciPost Phys. 5, 017 (2018).

[16] N. Goldman, G. Juzeliūnas, P. Öhberg, and I. B. Spielman, Rep. Prog. Phys. 77, 126401 (2014).

[17] M. Holthaus, J. Phys. B: At., Mol. Opt. Phys. 49, 013001 (2015).

[18] A. P. Itin and M. I. Katsnelson, Phys. Rev. Lett. 115, 075301 (2015).

[19] T. Mishra, A. Pallaprolu, T. Guha Sarkar, and J. N. Bandyopadhyay, Phys. Rev. B 97, 085405 (2018).

[20] L. C. Wang, X. P. Li, and C. F. Li, Phys. Rev. B 95, 104308 (2017).

[21] K. Plekhanov, G. Roux, and K. Le Hur, Phys. Rev. B 95, 045102 (2017).

[22] P. Roman-Taboada and G. G. Naumis, Phys. Rev. B 96, 155435 (2017).

[23] K. Takasan, A. Daido, N. Kawakami, and Y. Yanase, Phys. Rev. B 95, 134508 (2017).

[24] M. Thakurathi, D. Loss, and J. Klinovaja, Phys. Rev. B 95, 155407 (2017).
[25] M. Benito, A. Gómez-León, V. M. Bastidas, T. Brandes, and G. Platero, Phys. Rev. B 90, 205127 (2014).

[26] Z.-Z. Li, C.-H. Lam, and J. Q. You, Phys. Rev. B 96, 155438 (2017).

[27] X. Yang, B. Huang, and Z. Wang, Sci. Rep. 8, 2243 (2018).

[28] S. Saha, S. N. Sivarajan, and D. Sen, Phys. Rev. B 95, 174306 (2017).

[29] G. Jotzu, M. Messer, R. Desbuquois, M. Lebrat, T. Uehlinger, D. Greif, and T. Esslinger, Nature (London) 515, 237 (2014).

[30] K.-H. Ding, L.-K. Lim, G. Su, and Z.-Y. Weng, Phys. Rev. B 97, 035123 (2018).

[31] S. Owerre, Sci. Rep. 8, 4431 (2018).

[32] M. Hasan, D. Yudin, I. Iorsh, O. Eriksson, and I. Shelykh, Phys. Rev. B 96, 205127 (2017).

[33] A. A. Pervishko, D. Yudin, and I. A. Shelykh, Phys. Rev. B 97, 075420 (2018).

[34] D. Thuberg, E. Muñoz, S. Eggert, and S. A. Reyes, Phys. Rev. Lett. 119, 267701 (2017).

[35] J.-i. Inoue, J. Phys. Soc. Jpn. 87, 034711 (2018).

[36] K. Takasan, M. Nakagawa, and N. Kawakami, Phys. Rev. B 96, 115120 (2017).

[37] X.-X. Zhang, T. T. Ong, and N. Nagaosa, Phys. Rev. B 94, 235137 (2016).

[38] C.-K. Chan, Y.-T. Oh, J. H. Han, and P. A. Lee, Phys. Rev. B 94, 121106(R) (2016).

[39] P.-H. Fu, H.-J. Duan, R.-Q. Wang, and H. Chen, Phys. Lett. A 381, 3499 (2017).

[40] Z. P. Niu and X. Wu, Phys. Lett. A 382, 729 (2018).

[41] M. Ezawa, Phys. Rev. B 96, 041205 (2017).

[42] Z. Yan and Z. Wang, Phys. Rev. B 96, 041206(R) (2017).

[43] L. Bucciantini, S. Roy, S. Kitamura, and T. Oka, Phys. Rev. B 96, 041126(R) (2017).

[44] O. Deb and D. Sen, Phys. Rev. B 95, 144311 (2017).

[45] Z. Yan and Z. Wang, Phys. Rev. Lett. 117, 087402 (2016).

[46] D. Basov, R. Averitt, and D. Hsieh, Nat. Mater. 16, 1077 (2017).

[47] T. Oka and S. Kitamura, Annu. Rev. Condens. Matter Phys. 10, 387 (2019).

[48] J. H. Mentink and M. Eckstein, Phys. Rev. Lett. 113, 057201 (2014).

[49] J. Mentink, K. Balzer, and M. Eckstein, Nat. Commun. 6, 6708 (2015).

[50] J. Mentink, J. Phys.: Condens. Matter 29, 453001 (2017).

[51] M. Bukov, M. Kolodrubetz, and A. Polkovnikov, Phys. Rev. Lett. 116, 125301 (2016). 
[52] J. Liu, K. Hejazi, and L. Balents, Phys. Rev. Lett. 121, 107201 (2018).

[53] K. Hejazi, J. Liu, and L. Balents, Phys. Rev. B 99, 205111 (2019).

[54] M. M. S. Barbeau, M. Eckstein, M. I. Katsnelson, and J. H. Mentink, SciPost Phys. 6, 27 (2019).

[55] M. Eckstein, J. H. Mentink, and P. Werner, arXiv:1703.03269.

[56] J. B. Goodenough, Magnetism and the Chemical Bond (Interscience-Wiley, New York, 1963).

[57] D. Khomskii and G. Sawatzky, Solid State Commun. 102, 87 (1997).

[58] D. Khomskii, Phys. Scr. 72, CC8 (2005).

[59] Y. Tokura and N. Nagaosa, Science 288, 462 (2000).

[60] K. Kugel and D. Khomskii, Zh. Eksp. Teor. Fiz. 64, 1429 (1973) [Sov. Phys. JETP 37, 725 (1973)].

[61] S. V. Streltsov and D. I. Khomskii, Phys.-Usp. 60, 1121 (2017).

[62] O. E. Peil, M. Ferrero, and A. Georges, Phys. Rev. B 90, 045128 (2014).

[63] P. J. Phillips, X. Rui, A. B. Georgescu, A. S. Disa, P. Longo, E. Okunishi, F. Walker, C. H. Ahn, S. Ismail-Beigi, and R. F. Klie, Phys. Rev. B 95, 205131 (2017).

[64] A. S. Disa, D. P. Kumah, A. Malashevich, H. Chen, D. A. Arena, E. D. Specht, S. Ismail-Beigi, F. J. Walker, and C. H. Ahn, Phys. Rev. Lett. 114, 026801 (2015).

[65] S. H. Autler and C. H. Townes, Phys. Rev. 100, 703 (1955).

[66] See Supplemental Material at http://link.aps.org/supplemental/ 10.1103/PhysRevB.100.220403 for extra details of the ligand and metal-orbital schemes, which includes Refs. [80-82].
[67] L. Podobedova, D. Kelleher, and W. Wiese, J. Phys. Chem. Ref. Data 38, 171 (2009).

[68] J. Kanamori, Prog. Theor. Phys. 30, 275 (1963).

[69] C. Ballhausen and A. D. Liehr, J. Mol. Spectrosc. 2, 342 (1958).

[70] P. W. Anderson, Phys. Rev. 102, 1008 (1956).

[71] C. Naiman, J. Chem. Phys. 35, 323 (1961).

[72] A. D. Liehr and C. Ballhausen, Phys. Rev. 106, 1161 (1957).

[73] C. Ballhausen and A. D. Liehr, Mol. Phys. 2, 123 (1959).

[74] I. V. Solovyev, Phys. Rev. B 74, 054412 (2006).

[75] M. N. Iliev, M. V. Abrashev, H.-G. Lee, V. N. Popov, Y. Y. Sun, C. Thomsen, R. L. Meng, and C. W. Chu, Phys. Rev. B 57, 2872 (1998).

[76] M. N. Iliev, A. P. Litvinchuk, M. V. Abrashev, V. N. Popov, J. Cmaidalka, B. Lorenz, and R. L. Meng, Phys. Rev. B 69, 172301 (2004).

[77] D. M. Juraschek and S. F. Maehrlein, Phys. Rev. B 97, 174302 (2018).

[78] A. Subedi, A. Cavalleri, and A. Georges, Phys. Rev. B 89, 220301(R) (2014).

[79] M. Gu and J. M. Rondinelli, Phys. Rev. B 98, 024102 (2018).

[80] J. C. Slater, Phys. Rev. 36, 57 (1930).

[81] S. Sugano and R. G. Shulman, Phys. Rev. 130, 517 (1963).

[82] S. Y. Kim, T. Y. Kim, L. J. Sandilands, S. Sinn, M.-C. Lee, J. Son, S. Lee, K.-Y. Choi, W. Kim, B.-G. Park, C. Jeon, H.-D. Kim, C.-H. Park, J.-G. Park, S. J. Moon, and T. W. Noh, Phys. Rev. Lett. 120, 136402 (2018). 